\title{
Sinn und Unsinn von Indikatoren
}

\section{Die Bewertung von Indikatoren ist ein weit verbreitetes Instrument um Nach- haltigkeit zu messen. Indikatoren eigenen sich jedoch nur beschränkt zur Mes- sung von Nachhaltigkeit oder nachhaltiger Entwicklung. Ein systemischer An- satz würde sich besser eignen. Er wäre vollständiger und macht zusätzlich innere Widersprüche von reinen Indikatorenlisten deutlich.}

$\mathrm{N}$ Von Jasper Grosskurth achhaltigkeitsindikatoren sind ein beliebtes Werkzeug um Nachhaltigkeit in Zahlen auszudrücken. Diese Zahlen sollen uns zeigen, wie wichtig das Streben nach Nachhaltigkeit ist, ob wir uns in Richtung Nachhaltigkeit bewegen und was wir machen müssen, um nachhaltiger zu werden (1).

Für regionale Nachhaltigkeit ist diese Zielstellungen aber übertrieben. Der systemische Charakter regionaler Nachhaltigkeit verbietet es, aus der Analyse einzelner Teilaspekte, Schlussfolgerungen für Nachhaltigkeitsstrategien zu ziehen. Simulationsmodelle können einige der damit verbundenen Probleme lösen, werfen aber gleichzeitig neue auf. Neuartige qualitative Ansätze können möglicherweise zu neuartigen Indikatoren führen, die die systemischen Eigenschaften der Nachhaltigkeit mit einbeziehen.

\section{Die Suche nach der richtigen Nachhaltigkeit}

Seitdem Nachhaltigkeit auf wissenschaftlichen und politischen Tagesordnungen steht, wird versucht, sie zu definieren, zu messen, zu analysieren, zu kommunizieren und zu stimulieren. Leider eignet sich das Konzept zu all diesem nur beschränkt, was zu einer Debatte über Sinn und Unsinn von Nachhaltigkeit geführt hat. Nachhaltigkeit wegen dieser Schwierigkeiten zu verwerfen, hieße jedoch, das Kind mit dem Bade auszuschütten. Wenn wir versuchen regionale Nachhaltigkeit zu begreifen, sind vor allem drei Fragen wissenschaftlich und politisch relevant:

Was ist Nachhaltigkeit?

- Sind wir auf dem Weg zur Nachhaltigkeit?

- Was können wir tun, um Nachhaltigkeit zu erreichen?

Um diese Fragen zu beantworten wurden in der Vergangenheit lange Listen von Indikatoren und einige Indizes entwickelt. Stellvertretend will ich hier nur an die Bemühungen der OECD und des UN-DPCSD sowie an den Index of Sustainable Economic Welfare (ISEW) und den Ecological Footprint erinnern. Diese Ansätze unterscheiden sich in den zu Grunde liegenden Nachhaltigkeitsdefinitionen, das Prinzip der Ausarbeitung gleicht sich jedoch. Auf der Basis der Definition werden Teilaspekte der Nachhaltigkeit identifiziert. Für jeden Teilaspekt werden Indikatoren gewählt, die deren Entwicklung abbilden sollen. Ein typischer Teilaspekt ist die Umweltqualität. Daran zu koppelnde Indikatoren wären die Oberfläche von Naturgebieten oder die Anreicherung bestimmter Giftstoffe im Boden.

Der Vergleich von Indikatoren zwischen Regionen und deren Entwicklung lässt auf die Stärken und Schwächen einer Region im Bezug auf Nachhaltigkeit schließen. Indizes bündeln diese Information und vereinfachen so die Kommunikation, verschleiern aber Hintergründe. Die Antworten, die dieser Ansatz auf die oben genannten drei Fragen liefert, sind nicht ohne Schwierigkeiten.

\section{Transitionsstrategien zu einer nachbaltigeren Wirtschaft}

ist das Thema des Informationsdienstes

\section{Ökologisches Wirtschaften 2/04}

Wenn Sie potenzielle Beiträge haben, wenden Sie sich bitte an die Redaktion.
Die implizite Antwort auf diese Frage ähnelt sich in den oben genannten Beispielen. Eine Region ist nachhaltig, wenn keiner der gewählten Indikatoren Symptome von Nicht-Nachhaltigkeit aufweist. Was ein solches Symptom ist, ist in einigen Fällen explizit definiert. Im Falle des Ecological Footprint einer Region wäre ein Footprint, der größer ist als die tatsächliche Fläche der Region ein solches Symptom. In den meisten Fällen sind die Symptome aber frei zu interpretieren. So könnte eine Abnahme der Artenvielfalt ein Symptom fehlender Nachhaltigkeit sein, aber auch durch einen natürlichen, durchaus nachhaltigen Prozess verursacht sein.

Die Auswahl der Indikatoren basiert dabei fast nur auf inhaltlichen Kriterien. Für jeden Teilaspekt werden die inhaltlich am meisten deckenden Indikatoren gewählt. Einziges nicht-inhaltliches Kriterium ist die Möglichkeit der Quantifizierung. Das bedeutet, dass Teilaspekte, die nur schwer in Zahlen ausgedrückt werden können oder die schwer messbar sind von vorn herein ausgeschlossen werden. Beispiele sind soziale Spannungen oder politische Stabilität. In der Praxis führt dies unter anderem zu einer gestörten $\mathrm{Ba}$ lance zwischen ökonomischen, ökologischen und sozialen Aspekten.

Die Dispute über die quantitativen Aussagen (Symptome) lenken von der wichtigeren Diskussion ab, nämlich der zu den Systemeigenschaften, die nicht-nachhaltige Symptome verursachen, also den tatsächlichen Problemen. Dazu kommt, dass die ausgewählten Indikatoren bei diesem Ansatz bereits die Menge aller möglich zu erfassenden Symptome fehlender Nachhaltigkeit bilden. Wir suchen also Symptome nur dort, wo Licht ist, und lassen alle qualitativen Aspekte im Dunkeln. Damit schränken wir unsere Möglichkeiten signifikant und unnötig ein.

\section{Nähern wir uns der Nachhaltigkeit?}

Die Antwort auf die zweite Frage folgt aus der ersten. Wir bewegen uns in Richtung Nachhaltigkeit wenn die Indikatoren sich in die gewünschte Richtung entwickeln. Was gewünscht ist, ist entweder subjektiv oder normativ und hängt $\mathrm{ab}$ von der gewählten Definition von Nachhaltigkeit. Für viele Teilaspekte scheint das Gewünschte offensichtlich: nachhaltiger ist wohlhabender, sozial ausgeglichener und umweltverträglicher.

Schwieriger wird es, wenn man versucht, miteinander korrelierte Teilaspekte oder Indikatoren zu bewerten. Was ist die gewünschte Entwick- 
lung, wenn wir zwischen wirtschaftlicher Entwicklung und dem Erhalt von Ökosystemen wählen müssen, wie im Falle einer sehr profitablen, aber verschmutzenden Industrie? Bei Indikatorlisten stellt sich die Frage nicht, da die Zusammenhänge zwischen Indikatoren keine Rolle spielen. Nur mit einer Übersicht über die Teilaspekte und ihrer Zusammenhänge und nur mit Methoden zur Analyse dieser Übersicht ist es möglich um Probleme zu begreifen, statt lediglich Symptome zu identifizieren.

Die Antworten auf die ersten beiden Fragen werden oft verwendet um die Notwendigkeit zu Handeln zu untermauern. Eine sich auftuende Schere zwischen dem ISEW und dem Bruttoinlandsprodukt oder eine Reihe von Indikatoren, die sich entgegen der gewünschten Richtung entwickeln fordern eine Antwort auf die dritte Frage. Die dritte Frage erfordert ein tief greifendes Problemverständnis um nachhaltige Veränderungsprozesse in die Wege leiten zu können. Ausgehend von einer Liste von Indikatoren kann die einzige Antwort sein, dass Symptome fehlender Nachhaltigkeit verringert werden müssen. Als einzige Anknüpfungspunkte stehen uns dazu die identifizierten Indikatoren zur Verfügung. Das dahinter liegende System, und damit das Problem, kennen wir nicht. Eine Analogie bietet die industrielle Landwirtschaft. Ungeziefer, in diesem Kontext ein Symptom fehlender Nachhaltigkeit, wird mit Pestiziden bekämpft. Andere Symptome werden die Folge sein, dasselbe Symptom wird wieder auftreten, oft verstärkt und die vermeintliche Lösung wird dementsprechend hochgefahren. Das zu Grunde liegende Problem, die Ursache der Symptome, ist weder identifiziert noch gelöst.

In der Folge werden auch politische Interventionen nicht aus einem Systemverständnis heraus unternommen, sondern richten sich auf verstreute Symptome wie zum Beispiel Luftverschmutzung oder Staatsverschuldung. Dies führt zu Ineffizienz und oft unangenehmen Überraschungen. Ohne ein tief greifendes Systemverständnis, also der Einsicht in die Wechselwirkung eines Teilaspektes mit dem ihn umgebenden System, ist jede Intervention ein Glücksspiel. In der Vergangenheit wurden einige quantitative Modelle entwickelt, bei denen versucht wurde, Systeme abzubilden. Bekanntestes Beispiel ist das World 3 Model, auf dem das Buch „Die Grenzen des Wachstums” basiert (2). In diesen Modellen werden Indikatoren als Variablen eingesetzt. Die Simulation der Wechselwirkungen zwischen diesen Variablen erzeugt ein
Bild von Indikatoren, die sich in der Zeit in gewünschter, beziehungsweise ungewünschter Richtung verändern.

Interventionen sind in diesem Fall nicht beschränkt auf einzelne Indikatoren, sondern beziehen deren Systemumgebung mit ein. Dies ist ein Schritt voraus, auch wenn das zu Grunde liegende System nur auf Annahmen beruht. Leider beschränken die kommunizierten Ergebnisse dieser Modelle sich wiederum auf den Verlauf einzelner Indikatoren in der Zeit. Das angenommene System ist meist nur für Experten zu begreifen und eignet sich nicht für Kommunikation und Diskussion. Der Vorteil des besseren Systemverständnisses hat noch einen Preis. Die Restriktion auf quantifizierbare Einheiten wirkt sich für Simulationsmodelle noch restriktiver aus als bei Indikatorlisten. Denn nicht nur die Indikatoren selber, sondern auch ihre Zusammenhänge müssen, meist in Form von Differentialgleichungen, quantifizierbar sein.

\section{- Qualitative Systemanalyse: ein Schritt voraus?}

Um wissenschaftliches und politisches Nachhaltigkeitsverständnis zu erreichen, benötigen wir einen Ansatz, der es uns erlaubt, die grundlegenden Prozesse regionaler nachhaltiger Entwicklung zu begreifen und zu analysieren. Dieser Ansatz sollte transparent sein, wobei qualitative und quantitative Informationen kombinierbar sein sollten. Die Ergebnisse sollten die tatsächlichen Probleme nichtnachhaltiger Entwicklung und mögliche Anknüpfungspunkte für systemisch sinnvolle Lösungen hervorheben und einfach kommunizierbar sein. Am International Centre for Integrative Studies (ICIS) haben wir den Ansatz der Qualitativen System Analyse (QSA) entwickelt. QSA könnte als erster Schritt zu systemischen Indikatoren dienen, die die Grundlagen anstatt der Symptome nachhaltiger Entwicklung erfassen. QSA kombiniert und erweitert Methoden aus der Psychologie und der Socio-Kybernetik, deren Wurzeln in der Netzwerkund der Graphentheorie liegen. Anwendungen, auf denen QSA aufbaut, sind das SCENE Model und das Sensitivitätsmodell von Vester (3).

Der schrittweise aufgebaute QSA Ansatz beginnt mit dem Sammeln systemrelevanter Informationen. Diese Information wird in Form von Einflussdiagrammen wiedergegeben. Diese können mit Hilfe von netzwerkanalytischer Software interpretiert werden. Zusätzlich besteht die Möglichkeit, jede Form von Information, die sich entweder als Typologie oder auf einer Skala aus- drücken lässt, in den Analyseprozess mit einzubeziehen. So können zum Beispiel Unsicherheiten, Variabilität, Zeitdimensionen und der Einfluss verschiedener gesellschaftlicher Akteure auf Systemteile mit in die Analyse einfließen, ohne dass eine Quantifizierung notwendig wäre. Aber auch quantitative Informationen aus Simulationsmodellen oder Indikatorlisten können verarbeitet werden.

Die strategische Kombination dieser Information ergibt Hinweise auf effiziente Interventionspunkte, viel versprechende Strategien, und potenzielle Überraschungen. Der qualitative Charakter, bei gleichzeitig rigider Analyse vereinfacht den Brückenschlag zwischen wissenschaftlichem Denken und politischem Handeln. Projekte, in denen dieser Ansatz getestet und weiter entwickelt wird, laufen derzeit in der niederländischen Provinz Limburg und in den Niederlanden. Wir erwarten uns von diesen Projekten ein besseres Nachhaltigkeitsverständnis und Anregungen für bessere Interventionsmöglichkeiten, vor allem auf dem politischem Level, sowie neuartige Indikatoren.

\section{Anmerkungen}

(1) Vereinigung für ökologische Wirtschaftsforschung ( $\mathrm{Hg}$. ): Orte nachhaltiger Entwicklung. Reader zum 1. Netzwerkkongress junger Umweltsozialwissenschaftler. VÖWSchriftenreihe, Berlin 2003, englischsprachigen Publikation. des Autors: Grosskurth, J.: Sustainability indicators - why they mislead and what they should be like. In: Volkens, A./ Fischer, C./ Karmanski, A./ Bartelt, S./ Heinrichs, H. (Hg.): Orte Nachhaltiger Entwicklung: Transdiszipliäre Perspektiven. Schriftenreihe Vereinigung für ökologische Wirtschaftsforschung, Berlin 2003, pp. 44-49.

(2) Meadows, D. H./ Meadows, D. L. et al.: The Limits to Growth. New York 1972.

(3) Vgl. Grosskurth, J./ Rotmans, J.: The SCENE Model: getting a grip on sustainable development in policy making. In: Environment, Development and Sustainability, Elsevier, (accepted) sowie Vester, F.: Die Kunst vernetzt zu denken. München 2002.

\section{Der Autor}

Jasper Grosskurth arbeitet am International Centre for Integrative Studies (ICIS) an einer Doktorarbeit zum Thema regionale Nachhaltigkeit.

Kontakt: ICIS / UM, postbus 616, 6200 MD Maastricht, Niederlande. Tel. 0031-43-3882663, Fax 0031-43-3884916,

E-Mail: ja.grosskurth@icis.unimaas.n| 
(c) 20I0 Authors; licensee IÖW and oekom verlag. This is an article distributed under the terms of the Creative Commons Attribution Non-Commercial No Derivates License (http://creativecommons.org/licenses/by-nc-nd/3.o/), which permits unrestricted use, distribution, and reproduction in any medium, provided the original work is properly cited. 